Ebenfalls abkommandiert wurde Hitler, so Lüthy, zu einer politischen Beobachtung einer Versammlung der »neugegründeten >Deutschen Arbeiterpartei«, einer radikal-nationalistischen Vereinigung, »die damals wie Pilze aus dem Boden Münchens schoss, und mit der rednerischen Eroberung dieses `Spießervereins ` von sieben Mitgliedern begann Hitlers Welteroberung«, zumal Hitler nach »seiner ersten öffentlichen Rede vor 111 Zuhörern« feststellte: »Ich konnte reden! Nach dreißig Minuten waren die Menschen in dem kleinen Raum elektrisiert. $\ll^{60}$

\title{
1.5 Die nationalsozialistische Herrschaftspolitik bis 1938
}

Wie gesagt, ergab die empirische Auswertung der einzelnen Ausgaben des Monat, dass der Nationalsozialismus in 60 Beiträgen ausdrücklich Gegenstand war (unter Berücksichtigung sämtlicher Themenbereiche: „Vorgeschichte«, »Emigration«, »Intellektuelle« etc.). Abgesehen von Hofers Der »völkische Rechtsstaat«. Zur nationalsozialistischen Rechtspolitik (H. 125, S. 46-58), vereinzelten Bemerkungen und Einschätzungen von Trevor-Roper ${ }^{61}$ sowie Butlers Rezension zur Fritschkrise im Frühjahr $1938^{62}$ wurde in keinem weiteren Beitrag ausdrücklich auf die nationalsozialistische Herrschaft und Politik bis zum »Anschluss « Österreichs 1938 eingegangen. Insofern dieser Zeitraum der deutschen Geschichte thematisiert wurde, erfolgte die politische, historische oder wissenschaftliche Auseinandersetzung mit dem Nationalsozialismus in erster Linie »indirekt bzw. nebenbei« oder in quasi totalitarismustheoretischer Perspektive. ${ }^{63}$

Was man im Monat beispielsweise in Bezug auf die politischen Folgen »indirekt bzw. nebenbei« erfuhr, die mit dem historischen Datum des 30. Januar 1933, also der sogenannten Machtergreifung, verbunden war, war die Tatsache, dass nicht wenige tatsächliche politische Gegner des Nationalsozialismus, zum Beispiel (Links-)Intellektuelle, Schriftsteller, Künstler und deutsche Juden, die sich der drohenden persönlichen Gefahr gewahr wurden, Deutschland verließen und in die Emigration gingen.

60 Ebd., S. 159.

61 Siehe hierzu das Kap. II.6.8.

62 Rohan Butler, Der Fall Fritsch, in: Der Monat 4 (1952), H. 45, S. 313-316 (Rezension). Er teilt aufS. 313 mit, dass das Buch mit dem »einigermaßen schwerfälligen Untertitel >Die Fritsch-Krise im Frühjahr 1938 als Wendepunkt in der Geschichte der nationalsozialistischen Zeit« vom sogenannten Deutschen Institut für Geschichte der nationalsozialistischen Zeit herausgegeben wurde.

63 Siehe exemplarisch für die in quasi totalitarismustheoretischer Perspektive gemachten Ausführungen, den unter der Rubrik»Am Rande notiert« erschienenen Beitrag von H[ellmut] ][aesrich] in: Der Monat 11 (1958), H. 122, S. 85-87. Jaesrich berichtete zu Beginn davon, dass Boris Pasternak vom schwedischen Nobelpreiskomitee den »Nobel-Literaturpreis« zuerkannt wurde, allerdings von sowjetischer Seite, zumal vom sowjetischen Schriftstellerverband, unter Druck gesetzt bzw. »gezwungen« wurde auf den Nobelpreis zu verzichten (S. 85). Daran anschließend ging er unter der Überschrift Ein anderer Nobelpreis auf die näheren historischen Umstände der Verleihung des Friedensnobelpreises für den »deutschen Publizisten« Carl von Ossietzky im Jahre 1936 ein. Dort erfuhr man dann u. a., dass der parteiunabhängige Pazifist und Herausgeber der »linksgerichteten Weltbühne«, Ossietzky, $1933 \mathrm{im}$ Zusammenhang mit Verhaftungen im Kontext des »Reichstagsbrandes« in »verschiedenen Gefängnissen und Konzentrationslagern festgehalten« worden war und »im Mai 1938 starb« (S. 86). Daran anknüpfend stellte Jaesrich fest: »Der Name seiner Witwe schmückt heute das Titelblatt der äußerlich im alten Cewande wiedererstandenen Weltbühne in Berlin Ost«(S. 86 f.). 
Exemplarisch für einige Beiträge zu diesem Themenkomplex sollen im Folgenden zwei Veröffentlichungen genannt werden.

In einer der ersten Ausgaben des Monat, im Heft 11 vom August 1949, schrieb in der Rubrik »Musik« der New Yorker Musikschriftsteller Kurt List über Europäische Komponisten in Amerika. Ein Bericht über ihre Aufnahme und Weiterentwicklung. Dort erfuhr der Leser, dass zu den vielen Komponisten, die seit dem 19. Jahrhundert und in der ersten Hälfte des 20. Jahrhunderts von Europa in die Vereinigten Staaten ausgewandert waren, unter anderem Arnold Schönberg zählte, der im Alter von 59 Jahren 1933 nach Amerika einwanderte ${ }^{64}$ Auf Schönberg folgte 1934 der »linksgerichtete«, mit der Arbeiterbewegung in Deutschland sympathisierende, "politische Aktivist « und Komponist Hanns Eisler, der 1934 im Alter von 36 Jahren in New York ankam. ${ }^{65}$ Von den zahlreichen Komponisten, die neben Schönberg und Eisler vor dem Hintergrund eines »brutalen und oft terroristischen Nationalismus « ${ }^{66}$ nach Amerika gingen - zum Beispiel Kurt Weill -, war nach Ansicht von List, der 1937 in Amerika im Alter von 42 Jahren ankommende Paul Hindemith »der einzige Komponist, der wegen direkter politischer Angriffe Deutschland verlassen hatte ${ }^{67}$

Im September 1950 veröffentlichte der 1933 in die Emigration gegangene jüdischdeutsche Schriftsteller Hermann Kesten den Artikel Panorama. Eine literarische Betrachtung, den er mit folgenden Worten einleitete: "Die Deutsche Literatur wurde 1933 halbiert. Es begann die Epoche der Binnendichter unter der Diktatur des Schreckens und der emigrierten Dichter in der schreckensreichen Freiheit des Exils. ${ }^{68}$ Kesten thematisierte unter anderem das sogenannte Bild der deutschen Literatur im Exil und machte in diesem Zusammenhang die (nicht weiter quantifizierte) Feststellung, dass die Hälfte der emigrierten Literaten in den unterschiedlichsten Konzentrationslagern den Tod fand. Hierzu stellte er fest:

Sie starben auf der Flucht und in fremden Armeen. Sie starben durch Selbstmord und durch Krankheiten, Nachwirkungen des schweren Lebens im Exil. Wenn man die Zahl der im Exil gestorbenen mit der Zahl der in der Heimat gestorbenen deutschen Dichter vergleicht, so kommt man zu dem Schluß, daß das Exil gefährlicher und aufreibender gewesen sein muß als alle Schrecken der Heimat, samt Diktatur, Bombardierungen und Okkupation. ${ }^{69}$

$\mathrm{Zu}$ dieser Gruppe der nach 1933 emigrierten Schriftsteller zählten für Kesten auch Kommunisten, die insgesamt zwölf Jahre gegen die nationalsozialistische Diktatur gekämpft hatten und in zahlreichen Ländern aufgrund dieses Kampfes nicht nur

64 Siehe Kurt List, Europäische Komponisten. Ein Bericht über ihre Aufnahme und Weiterentwicklung, in: Der Monat 1 (1949), H. 11, S. 107-114, hier S. 108. Weiter hieß es über Schönberg auf S. 114, dass er in Amerika im Verlauf der Jahre vor dem Hintergrund der sogenannten »jüdischen Frage « und aufgrund des Einflusses »der allgemeinen Stimmung und der Schreckensnachrichten aus Deutschland eine immer radikalere politische Haltung« einnahm.

65 Ebd., S. 108.

66 Ebd., S. 111.

67 Ebd., S. 108.

68 Hermann Kesten, Panorama 1950. Eine literarische Betrachtung, in: Der Monat 2 (1950), H. 24, S. 592594, hier S. 592.

69 Ebd., S. 593. 
verfolgt wurden, sondern auch »durch Konzentrationslager, Gefängnisse und über Schlachtfelder gingen ${ }^{70}{ }^{70}$

Darüber hinaus wurde in den Beiträgen die für das einzelne Individuum in den allermeisten Fällen eine lebensgeschichtliche Zäsur darstellende Flucht resp. Emigration nach 1933 thematisiert. ${ }^{71}$ Neben dem oben bereits erwähnten Beitrag Der völkische Rechtsstaat. Zur nationalsozialistischen Rechtspolitik von Hofer aus dem Jahre $1959^{72} \mathrm{kam}$ im Monat lediglich Winfried Martini auf die innenpolitische Situation in Deutschland in den Jahren der NS-Machtkonsolidierung bis 1938 zu sprechen. ${ }^{73}$ Im Juni 1957 schrieb er unter der Rubrik »Hinweise ${ }^{74}$ aus zeitgeschichtlichem Anlass über die »Röhmaffäre« des 30. Juni $1934 .{ }^{75}$ Im Folgenden werden in nuce die historisch-politischen Kernaussagen und -interpretationen von Martini herausgearbeitet.

70 Ebd.

71 Siehe hierzu Franz Theodor Csokor, Ödön von Horváth, in: Der Monat 3 (1951), H. 33, S. 309-313, hier S. 311 f.; Charles Kessler, Ausdruck und Introversion. Deutscher Expressionismus und zeitgenössische amerikanische Malerei, in: Der Monat 4 (1951), H. 37, S. 91-95, hier S. 92; Hermann Kesten, Grenzler und Europäer. Gedanken über René Schickele, in: Der Monat 4 (1952), H. 45, S. 309-313, hier S. 311; ]ürgen Rühle, Arnold Zweig und die Kunst des inneren Vorbehalts, in: Der Monat 11 (1958), H. 123, S. 67-75, hier S. 68. Vgl. auch Hans Sahl, Welt in der Nußschale. Zu Richard Friedenthals InternierungslagerRoman, in: Der Monat 9 (1957), H. 100, S. 71-73 (hierbei handelte es sich um die Rezension von Richard Friedenthal, Die Welt in der Nußschale, München o. J.); Wolfdietrich Schnurre, Flucht in die Humanität. Zu Hans Sahls Zeitroman, in: Der Monat 12 (1959), H. 135, S. 75-77 (Rezension). Zur existentiellen Dimension bei Klaus Mann siehe Ludwig Marcuse, Das letzte Wort, in: Der Monat 4 (1952), H. 46, S. 408-410, hier S. 409f. (Rezension).

72 Walther Hofer, Der »völkische Rechtsstaat«. Zur nationalsozialistischen Rechtspolitik, in: Der Monat 11 (1959), H. 125, S. 46-58.

73 Die qualitative und quantitative Auswertung der einzelnen Hefte des Monat (H. 1 bis 139) ergab, dass in keinem Artikel ausdrücklich auf die nationalsozialistische Außenpolitik in den Jahren 1933 bis 1938 eingegangen wurde. In einem Beitrag kam die Zeitschrift indirekt auf die Rolle Deutschlands im Kontext der Genese des Spanischen Bürgerkrieges zu sprechen. Hierbei handelte es sich um den Beitrag: Werner Orlowsky, Das Vorspiel zum Kriege. Zum zwanzigsten Jahrestag des Spanischen Bürgerkrieges, in: Der Monat 8 (1956), H. 94, S. 22-30.

74 Obwohl der Beitrag in der keinesfalls zentralen Rubrik»Hinweise« veröffentlicht wurde und mithin im hinteren Bereich des Heftes abgedruckt wurde, kann vermutet werden, dass die Redaktion des Monat dem mehrere Seiten langen Artikel von Winfried Martini eine nicht geringe Bedeutung attestierte. Denn bereits auf der Umschlagseite der Ausgabe, d. h. der Heftnummer 105, wurde explizit auf den Artikel hingewiesen. So war dort zu lesen: »Winfried Martini: Zum 30. Juni 1934«. Auf der Innenseite derselben Ausgabe stand dann allerdings der eigentliche Titel Zelle 474 geschrieben.

75 Winfried Martini schreibt aus München: Zelle 474 ..., in: Der Monat 9 (1957), H. 105, S. 80-84. Martini schrieb den Artikel aufgrund des Prozesses vor der »4. Strafkammer des Landgerichts München I« gegen Sepp Dietrich, den damaligen »Kommandeur des SS-Wachbataillons Berlin, aus dem später die SS-Leibstandarte Adolf Hitler entstand« und gegen Michael Lippert (S. 80). In diesem Zusammenhang fragte sich Martini am Anfang seines Artikels, »welche strafrechtliche Rolle Sepp Dietrich am Spätnachmittag des 30. Juni 1934 gespielt [hat], als er [...] sechs SA-Führer im Hof der Strafanstalt München-Stadelheim auf Hitlers Befehl füsilieren ließ [...]? Und hatte einen Tag später Michael Lippert als SS-Sturmbannführer und Kommandeur der Dachauer KZ-Wachmannschaft gemeinsam mit SS-Brigadeführer Theodor Eicke, dem Kommandanten des Lagers, den Reichsminister Ernst Röhm, Stabschef des Obersten SA-Führers (der Hitler selbst war) in der Zelle 474 des gleichen Gefängnisses erschossen [...]?« Zu Martini vgl. Rudolf Augstein, Stimmzettel gegen Recht und Freiheit? Zu W. Martinis »Das Ende aller Sicherheit«, in: Der Monat 7 (1955), H. 76, S. 362-365 (Rezension). Er stellte 
Gleich zu Beginn des Artikels fällte Martini über den Angeklagten Sepp Dietrich das Urteil, dass es ziemlich schwerfällt,

ausgerechnet von Sepp Dietrich, den nun wirklich niemand für einen Erben Immanuel Kants halten kann, zu erwarten, er habe psychologisch und intellektuell auch nur die Möglichkeit gehabt, über die positiv-rechtliche oder gar moralische Legitimation des »Führerbefehls«, die sechs SA-Führer an die Wand zu stellen«, umständlich zu grübeln [...].

Für Martini war der »Allgäuer Dietrich«, der früh zum Hotelangestellten ausgebildet wurde und am Ersten Weltkrieg teilnahm, ein »Durchschnittsmensch ohne besondere amoralische Züge« (S. 80), sodass es glaubwürdig klinge, »als der kleine pyknische Mann seine Erschütterung andeutete, die er damals darüber empfunden hatte, daß er seine Kameraden, unter ihnen auch persönliche Freunde, erschießen lassen mußte« (S. 80). Und abschließend stellte Martini über den Angeklagten fest:

Ein Mann wie Dietrich war einer so hintergründigen und komplexen Situation, wie sie ihn am 30. Juni überfiel, in einem gewissen Sinne nicht weniger ausgeliefert als jene, die zu seiner Verwunderung und zu seinem Schrecken seine Opfer wurden; so klammerte er sich an eine dumpfe Interpretation der Pflicht, um, wenn er die Welt schon nicht mehr verstand, in ihr wenigstens die Orientierung nicht zu verlieren (ebd.).

Im Gegensatz hierzu beurteilte Martini den anderen Angeklagten Lippert als einen Menschen, dem man nur aufgrund der Kenntnis in Bezug auf seine "unheilige Verbindung mit Dachau « zutrauen konnte, dass er mit »seinem Kumpanen Eicke, im Rahmen der Zellentür stehend, den wehrlosen Röhm erschoß« (S. 81). Vor dem Hintergrund des (Gerichts-)Urteils war der Autor des Monat der Ansicht, dass »die Umstände« (für die beiden Angeklagten Dietrich und Lippert) durchaus die Vorstellung begreiflich gemacht hätten, »es habe ein Staatsnotstand bestanden«, so

daß aber nach vollzogener Verhaftung der wichtigsten SA-Führer die Annahme, sie müßten ohne Urteil auch noch getötet werden, offensichtlich zu abwegig gewesen sei, um irgendeine Berücksichtigung zu verdienen. Also war die Rechtswidrigkeit des »Füh-

zuallererst fest, dass Martini »schon im Titel der parlamentarischen Demokratie den Vorwurf macht, sie könne die Sicherheit der ihr anhängenden Bürger im Zeitalter der Krisen nicht mehr gewährleisten, um so weniger, als die ihr wesenhafte Widersprüchlichkeit offen zutage liege« (S. 362). Und weiter in Bezug auf den »Hitler-Staat«: Dieser »war eine seigentliche< Demokratie, wurzelnd in der intoleranten Tradition Rousseaus und der Jakobiner, aber er war das Gegenteil eines Rechtsstaates. In Krisenzeiten, sagt Martini, wird die >demokratische Komponente der parlamentarischen Demokratie den Rechtsstaat unterkriegen. [...] Aber Martini greift nicht zu. Er will nicht untersuchen, wie die Demokratie noch funktionieren kann, er hat sich vorgenommen, zu beweisen, daß sie nicht mehr funktionieren kann, und er beweist es. [...] Auch daß die Republik von Weimar die Probe Hitler nicht bestanden hat, ist noch kein Beweis gegen die parlamentarische Demokratie. Die Probe Hitler haben die Demokratien und Diktaturen der Welt insgesamt schlecht genug bestanden. Ein Hitler in all seiner Banalität war eine Ausnahmeerscheinung, gegen die Regierungsformen und -systeme nicht geeicht werden können« (S. 363 f.). In seinem abschließenden Resümee schrieb Augstein u. a.: »Martini hat seinem Buch den Untertitel gewählt >Eine Kritik des Westens< [...], aber es hilft dem Westen nicht, zu erfahren, daß er die falsche Staatsform hat« (S. 365). 
rerbefehls« nicht nur objektiv klar, sondern mußte von den Angeklagten auch durchschaut oder zumindest als Problem erkannt werden? Die Frage ist,

so Martini weiter,

ob jemand, der sich die psychologische Möglichkeit bewahrte, »Führerbefehle« einer rechtlichen und moralischen Analyse zu unterwerfen, überhaupt ein Nationalsozialist war. Konnte man aber von Nationalsozialisten die Fähigkeit erwarten, aus dem Wertesystem, dem sie sich nun einmal ergeben hatten und zu dem gerade auch die Vorstellung vom unfehlbaren Führer-Messias gehörte, einfach heraustreten? (Ebd.).

Martini war überzeugt davon, dass die SA am 30. Juni 1934 keineswegs die Absicht hatte, einen »Putsch« durchzuführen. Denn auch aufgrund der »Vorarbeiten« für den oben genannten Prozess seitens des Münchener »Instituts für Zeitgeschichte« war es für ihn »völlig ausgeschlossen«, dass ein Putsch beabsichtigt war (S. 82). Dies ergab sich durch die damaligen historischen Bedingungen: denn auch wenn ein »Putschplan« der SA vorgelegen hätte, wäre es nicht zuletzt infolge der "miserablen« Bewaffnung insbesondere für die Reichswehr kein »ernsthaftes militärisches Problem« gewesen, ebendiesen »Putsch « niederzuschlagen, und: »nur die Reichswehr aber wäre - auch das war schon damals klar - der militärische Adressat des Putsches gewesen« (ebd.).

$\mathrm{Zu}$ den Verhafteten des »30. Juni« zählten nach Auskunft des Autors »der Chef des Politischen Amtes der Obersten SA-Führung in der Berliner Tiergartenstraße«, der Gruppenführer von Detten und dessen Stellvertreter, der SA-Oberführer Hans von Falkenhausen, die beide in Berlin »in der Nacht vom 1. zum 2. Juli in Lichterfelde erschossen« wurden. Der »Initiator ihrer Erschießung«, so die Ansicht von Martini, »sei Goebbels gewesen [...]. Beide waren aus dem >Stahlhelm $<$ übernommen worden, beide waren echte Konservative [...], beide hatten wenig für Seldte übrig und standen dem >Dritten Reich`voller Reserve und Skepsis gegenüber« (ebd.). Wie gesagt, stand für Martini zweifellos fest, dass der SA-Führer Ernst Röhm zum 30. Juni 1934 keineswegs den Plan hatte zu putschen. Dazu Martini: „Ob er sich überhaupt mit dem Gedanken trug, sehr viel später einen zu wagen, wird sich kaum mehr feststellen lassen. Daß er Hitler gegenüber nicht die Gefühle sklavischer Unterwürfigkeit kannte, weiß man: er äußerte sich oft genug spöttisch und verächtlich über den >lächerlichen Gefreiten<, die >Primadonna« (ebd.). Gleichwohl, so der Autor, gab es in der SA die verbreitete Vorstellung bzw. die »entsprechende Stimmung « für eine sogenannte zweite Revolution (S. 83).

Mit Blick auf die Revolution im Allgemeinen konstatierte Martini, ${ }^{76}$ dass sich $» j e d e$ Revolution« nach der »Stunde des Sieges« spaltet. »Die eine >Aufbaugruppe versteht jenes Gesetz dank der praktischen Berührung mit der Macht, sie relativiert das >radikal Neue oder verschiebt seine volle Verwirklichung auf später. Die andere dagegen«, so Martini weiter,

76 Martini zitiert hier Karl Mannheim, 1893 in Budapest geboren und im April 1933 aus Deutschland, über Amsterdam nach London, emigrierter jüdischer Soziologe, der u. a. mit der 1929 erschienenen Studie Ideologie und Utopie bekannt wurde, der einmal bemerkte, »daß sauch das radikal Neue am Ende nur eine Komponente der späteren Wirklichkeit zu sein pflegt, die ja immer mehr Kräfte und Strömungen zu verarbeiten hat als gerade jene eine, an der einer bestimmten Aufbaugruppe am meisten gelegen ist،.«(Martini, Zelle 474 ..., S. 83) 
fühlt sich dem »radikal Neuen«, das in den Kampfjahren beide Gruppen gemeinsam und in bruchloser Front angestrebt hatten, nach wie vor ausschließlich verpflichtet, es darf nicht nur »eine Komponente der späteren Wirklichkeit« sein, sie spürt nicht den vielfältigen Zwang zur Anpassung und daher Abweichung, den die Dinge selber auf die der Macht am nächsten stehende Gruppe ausübt. Sie hält vielmehr die Abweichung für den Ausfluß boshaften »Verrats«, sie wird nun zur Bannerträgerin des »wahren« Kerns der Bewegung, kurz, sie wird zum »Trotzkismus«, den jede Revolution, wenn auch in höchst verschiedenen Formen und mit höchst verschiedenen Antrieben, so doch unweigerlich und aus ihren eigenen Reihen erzeugt. ${ }^{77}$

Nach Ansicht des Autors war im >Dritten Reich« Röhm »der Träger« ebendieses sogenannten Trotzkismus, er fühlte sich als Gralshüter des >wahren Nationalsozialismus, obwohl er bis zu seinem Tode, so das Urteil Martinis, »überzeugter Monarchist geblieben war, er spielte mit dem Gedanken einer `Zweiten Revolution`, die dem >wahren Nationalsozialismus zum Durchbruch verhelfen sollte, er hoffte sie mit Hitler zu verwirklichen, notfalls ohne ihn « ${ }^{78}$. Für Martini stand das Wort einer >permanenten Revolution`als Ausdruck für eine Enttäuschung, genauer gesagt: für eine

naive Enttäuschung darüber, daß mit der staatlichen Macht ein retardierendes Moment in die »Bewegung« gekommen war, die nun nicht mehr bewegt und beweglich genug erschien. So sollte die »permanente Revolution«jenem eigentümlichen Element des Nationalsozialismus entsprechen, für das die »Bewegung «als der unentwegte, sich unentwegt steigernde und rauschhafte Cenuß der kollektiven nationalen Vitalitäten paradoxerweise zugleich auch das eigentliche Ziel war. ${ }^{79}$

Die selbstgestellte Frage, warum insbesondere Röhm zum »Träger des NS->Trotzkismus« "geworden war und in welchen tatsächlichen Forderungen »dieser seinen Ausdruck fand «, versuchte Martini insbesondere auf der Basis der Veröffentlichung Die "Zweite Revolution« - Der 30. Juni 1934 von Hermann Mau, die 1953 in der vom Münchener Institut für Zeitgeschichte herausgegebenen Zeitschrift Vierteljahreshefte für Zeitgeschichte erschien ${ }^{80}$ und die nach seinem Dafürhalten eine "glänzende Arbeit« war, die »die wesentlichen Ursprünge, Gründe und Hintergründe des schauerlichen Dramas dargestellt und analysiert« hat. Nach Auffassung von Martini verdankte

die NSDAP ihren Aufstieg nicht zuletzt der Idee Röhms, die Partei, die sich organisatorisch im herkömmlichen Rahmen bewegte, mit dem Wehrverband der SA zu kombinieren: erst diese Verbindung stellte die »Bewegung« dar, das absolut Neuartige, das schließlich den Erfolg brachte. Die Kampfzeit erlaubte allen Beteiligten die Fiktion von der Einheit der Bewegung, man gab sich keine Rechenschaft über die tiefen Unterschiede, die zwischen Partei und Wehrverband nach soziologischer Struktur und immanenten Zielen bestanden. Erst die »Machtergreifung«brachte die Unterschiede

77 Ebd., S. 83.

78 Ebd.

79 Ebd., S. 84

80 Hermann Mau, Die »Zweite Revolution«-Der 30. Juni 1934, in: Vierteljahresheft für Zeitgeschichte 1 (1953), S. 119-137. 
zum Bewußtsein und zur Wirkung. Was sollte nun die Aufgabe der SA sein, vor allem, nachdem der blutige Terror, den sie in den ersten Monaten unter dem Namen »hilfspolizeilicher« Dienste ausgeübt hatte, teils reduziert, teils in andere Hände übergegangen, jedenfalls ihr entglitten war? ${ }^{81}$

Nach Meinung von Martini wollte die "NS-Staatsführung« der SA keine Aufgaben mehr einräumen, und zwar aus dem Grund, dass sie »mit ihr nichts mehr anzufangen wußte«. Vor diesem Hintergrund war es »nur natürlich und konsequent, daß Röhm, der sich stets als Soldat fühlte, nun den eigentlichen Sinn des Wehrverbandes verwirklichen wollte«. Was nämlich Röhm vorschwebte, war aus der einstmals knapp 300 ooo Mann starken SA, die Anfang 1934 auf schätzungsweise 4,5 Millionen angewachsen war, »eine Miliz zu machen, die der Reichswehr nicht unterstellt werden sollte $\ll^{82}$. Und genau diese Vorstellungen mussten aus der Sicht des Autors für die Reichswehr besorgniserregend sein. »Hitler aber«, so Martini,

hatte kein Interesse daran, daß gerade unter Röhm, der es als einziger an Popularität mit ihm aufnehmen konnte, sich eine neue Macht bildete, und zugleich glaubte er seine Rüstungspläne in den Händen der Fachleute aus der Bendlerstraße weitaus besser als in denen von der SA-Führung aufgehoben: so zog Röhm sich die Gegnerschaft sowohl der politischen wie der militärischen Reichsführung zu. ${ }^{83}$

Die »Hochspannung vom Frühjahr 1934« musste für Martini keineswegs »zu einem Putsch« bzw. »zu jenem Massenmord « führen. Aber »einige Leute«, so beurteilte er die damalige Situation, hatten - aus ganz unterschiedlichen Motiven - »ein Interesse daran, die Spannung durch Lancierung alarmierender Nachrichten, die auch die zuständigen Stellen der Reichswehr passierten, bis zu dem Punkt zu erhitzen, daß die SS unter - teilweise gutgläubiger - Assistenz der Reichswehr losschlagen konnte«. Und in diesem Fall stand für ihn fest, dass Göring, Goebbels, Himmler und Heydrich »auf der braunen Seite mit Sicherheit zu denen [gehörten], die die furchtbaren Karten mischten: wieweit sie Hitler schoben, wieweit er selber eine bewegende Kraft war das wird sich kaum mehr exakt ausmachen lassen ${ }^{84}$.

Zum Abschluss seines Zeitschriftenartikels Zelle 474... zitierte Martini den im Strafprozess gegen Sepp Dietrich und Michael Lippert als Zeuge aufgetretenen Rudolf Diels - »der 1933 unter Göring die Abteilung I A des Berliner Polizeipräsidium zum preußischen Geheimen Staatspolizei-Amt entwickelt hatte« (S. 80) -, der »im 30.

81 Winfried Martini (schreibt aus München): Zelle 474 ..., in: Der Monat 9 (1957), H. 105, S. 84.

82 Ebd.

83 Ebd.

84 Ebd. Aufseiten der Reichswehr war für Winfried Martini Reichenau »die wissende und zugleich treibende Kraft«, der einerseits den in die »Röhm-Affäre« eingeweihten »schwachen Blomberg« manipulierte und andererseits »anscheinend auch Schleicher freigegeben hat [...].« Neben Reichenau und Blomberg waren nach Ansicht Martinis aller Voraussicht nach »alle beteiligten Reichswehrstellen gutgläubig gewesen « und er konstatierte - unter Berufung auf den o. g. Aufsatz von Hermann Mau-, »daß das Spiel >offenbar schon von Leuten im Range von Fritsch und Beck nicht mehr durchschaut wurde«. Allerdings, so der Autor, überschätzte Reichenau »die Rolle, die er spielte: denn auch er wollte kaum die Früchte, die der Massenmord später trug, auch er diente den schwarzen Beelzebuben, mit denen er die braunen Teufel auszutreiben wähnte, nur als halbwissendes Werkzeug«(S. 84). 
Juni den letzten Schub zum Verhängnis [sah]; er bedeutete die Einführung des Mordes als staatliches Prinzip«. Denselben Sachverhalt brachte Martini zufolge Mau in seinem Aufsatz Die »Zweite Revolution« - Der 30. Juni 1934 zum Ausdruck, der nämlich schrieb: "Es ist von nun ab in der nationalsozialistischen Politik, als seien gewisse Sicherungen durchgebrannt. Macht und Gewalt haben sich unwiderruflich miteinander verbunden. $\ll^{85}$

Da der Beitrag von Martini neben dem angesprochenen Aufsatz von Hofer die einzige Veröffentlichung im Monat war, der die Phase der unmittelbaren Machtkonsolidierung des Nationalsozialismus thematisierte, d. h. das Jahr 1933 und vor allem die erste Hälfte des Jahres 1934, und zudem versuchte, die historisch-politischen Hintergründe der »Röhmaffäre « einer substanziellen Deutung zu unterziehen, wurde dieser Beitrag ausführlich referiert. Denn wie gesagt, anhand der einzelnen Hefte des Monat erfuhr man im Grunde genommen kaum etwas über die Jahre 1933 bis 1938. Das galt sowohl für die deutsche Außenpolitik als auch für die Innenpolitik und infolgedessen für die »udenpolitik« des nationalsozialistischen Herrschaftssystems. Die Außenpolitik in diesem Zeitraum war im Grunde genommen ein Anathema, und auf die nach der »Machtergreifung« in Gang gekommene (Terror-)Politik gegenüber Kommunisten, Sozialdemokraten und der jüdischen Bevölkerung kam der Monat nur »nebenbei« zu sprechen. Einzelheiten über die nach der nationalsozialistischen Machteroberung und -konsolidierung in Gang gekommene »Judenpolitik« des »Führerstaates« erfuhr man nur vor dem Hintergrund der genauen Lektüre der einzelnen Ausgaben des Monat. Hierbei wurde in allererster Linie das Jahr 1938 fokussiert.

So hieß es beispielsweise bereits im dritten Heft, d. h. im Dezember 1948, bei Lasky in der Besprechung des Buches Vom Andern Deutschland. Aus den nachgelassenen Tagebüchern 1938 bis 1944 von Ulrich von Hassell: "Diese Tage<, verzeichnete er im Winter 1938, sstehen unter der von allen anständigen Menschen schwer empfundenen Last der beschämenden Vorgänge (Pogrom) im November [...].« ${ }^{86}$ Edouard Roditi wiederum kam im Rahmen seines unter der Rubrik »Bildende Kunst « erschienenen Artikels Der Leidensweg der Berliner Museen im Juni 1949 auf die antijüdische Politik des Nationalsozialismus im Jahre $1938 \mathrm{zu}$ sprechen. ${ }^{87}$ Vor dem Hintergrund eines von der NSDAP bzw. von Hitler 1937 »unterzeichneten Befehls«, die Berliner Nationalgalerie »von aller sogenannten >entarteten Kunst $\mathrm{zu}$ `säubern ${ }^{8},{ }^{88}$ schrieb Roditi:

\footnotetext{
85 Zit. n. ebd., S. 84.
}

86 M[elvin] ]. L[asky], v. Hassell und die Fäulnis im Staate, in: Der Monat 1 (1949), H. 3, S. 99-102, hier S. 100 (Rezension).

$87 \mathrm{Zu}$ den Folgen der antijüdischen Politik des NS-Regimes siehe auch Alfred Polgar, Der Crosse Dilettant. Zu Egon Friedells »Kulturgeschichte der Neuzeit«. Der Mann und das Werk, in: Der Monat 2 (1950), H. 16, S. 410-419. Hier war auf S. 413 von dem Remigranten Alfred Polgar zu lesen, dass sich der in Österreich geborene jüdische Philosoph, Journalist, Schauspieler und Schriftsteller Egon Friedell, wenige Tage »bevor Österreich im nationalsozialistischen Wolfsrachen verschwand«, seinen Selbstmord angekündigt hatte und (sich aller Wahrscheinlichkeit nach) »am 14. März 1938 nachts, als er SSMänner ins Tor seines Hauses treten sah«, im Schlafrock »aus dem Fenster«stürzte.

$88 \mathrm{Vgl}$. hierzu auch H[ans] H[einz] Stuckenschmidt, Urbanität und Volksliedgeist. Über Béla Bartók, in: Der Monat 5 (1953), H. 59, S. 512-517, wo aufS. 513 darüber berichtet wurde, dass bereits 1936 »in Hitlerdeutschland, in Düsseldorf, eine berüchtigte Ausstellung, >Entartete Musik«, gezeigt« wurde. Nachdem Bartók erfuhr, dass seine Werke in der Ausstellung fehlten, protestierte er, so Stuckenschmidt, in einem Brief an das »Ribbentrop-Ministerium« entschieden dagegen. »Den deutschen Faschismus 
Im Jahre 1938 unternahm der nationalsozialistische Staat weitere Aktionen gegen die in Deutschland befindlichen Kunstwerke. Am 9. Und 10. November wurde ein organisierter Pöbel aufgerufen, angeblich »jüdischen Besitz« zu plündern und zu zerstören. Dabei wurden z.B. deutsche Plastiken der Gotik und Renaissance vernichtet, die von ihrem nichtjüdischen Besitzer bei dem jüdischen Kunsthändler Bernheimer in München zum Verkauf ausgestellt worden waren. Zahllose Kunstwerke, die sich zufällig in jüdischen Häusern oder Ceschäften befanden, wurden zerstört oder beschlagnahmt. Die SS plünderte in gleicher Weise die Berliner Freimaurerlogen aus, und das Museum der Berliner jüdischen Gemeinde in der Oranienburger Straße hörte zu bestehen auf. ${ }^{89}$

Auf den nationalsozialistischen Pogrom am Abend des 9. Novembers 1938 gegen die Juden in Deutschland kam neben Rudolf Hagelstange in seinem Beitrag Sühne und Ehre auch Hellmut Jaesrich im Rahmen seiner Besprechung der Tagebücher von Jochen Klepper im Jahre $1957 \mathrm{zu}$ sprechen. Hagelstange schrieb angesichts seiner persönlichen Erfahrungen, die er mit einigen Freunden in Erfurt am 9. November 1938 machte, unter anderem:

Als wir ankamen, war der Himmel gerötet. Bestellter uniformierter Mob hatte Feuer an die Synagoge gelegt und sich dann, berauscht an diesem Fanal, über die Wohnungen der jüdischen Mitbürger hergemacht. Wie sollte, der die Wohnung Cottes schändet, vor dem Hause des Menschen Ehrfurcht hegen! In dieser Nacht wußte ich, daß wir verurteilt waren - nicht vor einem menschlichen Cericht. ${ }^{90}$

Und hinsichtlich der »deutschen Ehre« hieß es eingedenk des nationalsozialistischen millionenfachen Mordes an »wehrlosen jüdischer Menschen« (S. 585) bei Hagelstange in diesem Zusammenhang weiter:

Was unserer Ehre aber zweifellos zum abträglichsten geschah, das war das mit dem Bodensatz von längst widerlegten Ideen und Thesen - ich meine den »Arier«- begründete Massaker an den Juden deutscher und vor allem fremder Staatszugehörigkeit. Als es mit jenem 9. November 1938 eingeleitet wurde, öffentlich, vor den Augen der Mitbürger, habe ich [...] in viele entsetzte Augen geblickt, habe bestürzte Reden gehört, Zweifel, Empörung und Widerspruch, selbst von jenen, die Hitler für ihren Mann hielten und einen seiner braunen Röcke oder sein Abzeichen trugen. Und die Fama wurde dann allzu begierig aufgesogen: $e r$ habe dies alles nicht gewollt. Es sei ein Übergriff untergeordneter Stellen gewesen. Das Gefühl für Recht und Menschlichkeit war nicht erstorben $[. ..] .^{91}$

Der Mitherausgeber des Monat, Hellmut Jaesrich, kam auf den NS-Pogrom anlässlich von Jochen Kleppers Tagebucheintragungen aus den späten 1930er-Jahren zu sprechen,

kennzeichnete er als sein Räuber- und Mördersystem`, den Rassen-Fragebogen der Wiener AKM, einer Autorenschutzgesellschaft, ließ er unbeantwortet«.

89 Edouard Roditi, Der Leidensweg der Berliner Museen, in: Der Monat 1 (1949), H. 8/9, S. 141-145, hier S. 142. Zur Beschlagnahmung von Kunst- und Kulturgütern aus den Berliner Museen durch die »sowjetische Militärregierung im Jahre 1945«siehe S. $143 \mathrm{ff}$.

90 Hagelstange, Sühne und Ehre, S. 585.

91 Ebd., S. 587. 
wo dieser im Kontext der in den "neuen Kirchengesetzen" enthaltenen sogenannten Arierparagrafen auch auf die >Widerstandsbewegung ‘ gegen antijüdische Tendenzen bzw. Maßnahmen im Bereich der Literatur einging. Bei Jaesrich war dazu zu lesen:

[E]ine sichtbare geistige Resistance [konnte sich] auch nur dort ansiedeln, wo Staat und Partei im Interesse irgendwelcher ihnen genehmer Werte ein Auge zudrückten. So wurde das Buch vom Soldatenkönig für die Wehrmacht in größeren Partien gekauft, ungeachtet der Tatsache, daß sein »jüdisch versippter « Autor nur dank einer zeitlich begrenzten Sondergenehmigung der Reichsschrifttumskammer überhaupt publizieren durfte, unbeschadet der Tatsache auch, daß es für jeden, der Ohren hatte zu hören, in ganz anderen Tönen als den erwünschten sprach. So gefiel »Der Vater« Rudolf Alexander Schröder und Rudolf Pechel, gefiel aber auch Hans Hinkel und Reichsinnenminister Frick. Doch weder das noch die unzähligen Interventionen, die Telefonate, Besuche, Briefe, über die Klepper genaueste Buchführung ablegt, konnten das Zuschnüren der Arisierungsschlinge aufhalten, die sich seit dem 9. November 1938 und erst recht seit Kriegsbeginn immer schneller zuzog. ${ }^{92}$

\subsection{Der Zweite Weltkrieg und der deutsche Widerstand}

Der mit Abstand größte Teil der Veröffentlichung zur Geschichte des >Drittes Reiches im Monat bezog sich auf den für Deutschland nur wenige Jahre zuvor mit einer totalen militärischen und politischen Niederlage zu Ende gegangenen Zweiten Weltkrieg. Einen zweiten Schwerpunkt bildete in diesem Kontext der konservative militärische Widerstand gegen den immer wieder als "Hitlerstaat« apostrophierten Nationalsozialismus, der in erster Linie vonseiten einer Minderheit aus der deutschen Militärelite erfolgte. Auch weil in einigen Beiträgen der enge Zusammenhang zwischen dem Zweiten Weltkrieg und dem »deutschen Widerstand « fokussiert und angesprochen wurde, werden in diesem Abschnitt beide Themenbereiche gemeinsam behandelt. Abgesehen von den Beiträgen, die quasi in totalitarismustheoretischer Perspektive geschrieben wurden, untersuchte und thematisierte Der Monat den Zweiten Weltkrieg (sowie dessen unmittelbare Vorgeschichte) explizit und implizit in 22 Beiträgen, den »deutschen Widerstand « in elf Beiträgen..$^{93}$ Die Auswertung der einzelnen Ausgaben des Monat ergab, dass auf den facettenreichen, komplexen sozialdemokratischen und kommunistischen (Partei-)Widerstand ${ }^{94}$ (wie z.B. von Löwenthal oder Borkenau) sowie auf den individuellen bzw. organisierten jüdischen Widerstand (wie z. B. von

92 Jaesrich, In Qualen gelebt, S. 73

93 Hierbei wurden auch Beiträge berücksichtigt, die nicht ausdrücklich den militärischen und politischen Kontext des Zweiten Weltkrieges untersuchten und thematisierten, sondern bereits auch auf den deutschen bzw. christlichen Widerstand im Verlauf der 1930er-Jahre eingingen. Siehe hierzu bes. Jaesrich, In Qualen gelebt, S. 73.

94 Der Begriff »Widerstand« wird hier verstanden als nicht ausschließlich militanter, d. h. militärischpolitischer Kampf gegen den Nationalsozialismus, sondern auch als eine Auseinandersetzung, die gewissermaßen eine politisch-intellektuelle und politisch-humanistische Form annehmen konnte, indem man in Zeitungs-, Zeitschriften- oder auch Buchveröffentlichungen gegen »Hitlerdeutschland « kämpfte; oder indem man auf wissenschaftlichen Symposien - wie zum Beispiel Hans Kohn Forschungsarbeiten zur Analyse des Nationalsozialismus präsentierte, oder indem man - wie zum Beispiel Hannah Arendt - in jüdischen (Flüchtlings-)Bewegungen arbeitete. 